\title{
Investigation of the Fit of Computer-based Parametric Garment Prototypes
}

\author{
Maja Mahnic*, Slavenka Petrak \\ University of Zagreb, Faculty of Textile Technology, Prilaz baruna Filipovića 28a, Zagreb, Croatia
}

\begin{abstract}
The paper presents part of a comprehensive and complex investigation in the field of computer design and 3D garment simulation within which it investigates developed computer-based parametric prototype of a men's suit and whether it is suitable for computer adaptation according to measurements of different body characteristics. The investigation was conducted by segments. It was necessary to do tests and measurements using innovative computing technologies and appropriate measuring equipments. Using the $2 \mathrm{D} / 3 \mathrm{D}$ CAD system for the preparation of garment construction, a $2 \mathrm{D}$ pattern was developed for the purpose of computer grading for selected sets of sizes according to different figures and statures. The scanning data process was performed through using a 3D laser scanner and a computer analysis was made with anthropometric measurements of the body on a sample of male subjects of different anthropometric body characteristics. The most complex part of the research was finding mathematical expressions and comprehensive development of a men's suit model with the aim of translating 2D pattern parts from vector into parametric format, which allows simultaneous changes in several corresponding segments of pattern pieces.
\end{abstract}

Keywords: Computer Garment Construction; CAD System; 3D Simulation; Made-to-measure

\section{Introduction}

The area of scientific research related to the development of 3D CAD systems for garment simulation has been extensively developed for the last two decades. In the initial phase of the development, efforts of researchers focused on the research of computer simulation of garments based on the physical-mechanical properties of fabrics and the intention to achieve the best possible realistic simulations of garments [1-3]. Development and application of 3D body scanners and linking of scanner with CAD systems for construction and simulation of clothing enabled further development of systems and methods for the simulation of clothing adjusted according to individual anthropometric measurements [4-7]. Recent studies have been focused on the development of CAD systems for virtual try-on, fitting evaluation and style editing with a propose

\footnotetext{
${ }^{*}$ Corresponding author.

Email address: maja.mahnic@ttf.hr (Maja Mahnic).

Guest editor: Budimir Mijovic
} 
to speed up clothing design and pattern development process [8-13]. Many studies are also focused on the investigation and classification of human body types [14-18] as a starting point for a mass customization garment production [19-23]. On today's market there is a large number of commercial CAD systems that enable a comprehensive development of patterns across all phases of construction preparation and simulation of garments for the purpose of making prototypes of computer garment models. In this context, a significant contribution has been made to the development in the field of clothing engineering and the preparation for garment construction in the garment industry. However the implementation of the concept of the industrial production of clothes made to measure based on the application of modern computer technologies haven't been accepted in real terms, except in a small number of famous garment manufacturers in developed Western countries. The reasons for this should be sought in the complexity of an integrated concept that includes the implementation of highly sophisticated computer and measuring equipment in real conditions of production preparation. In this connection significant financial investments are necessary for the implementation and maintenance of such systems. As a particular aggravating circumstance the complexity of the whole preparation includes a systematic approach and combining knowledge from several scientific disciplines. In this context it is necessary to systematically connect the knowledge of the anthropometric measurements of the body, objective evaluation of mechanical fabric properties, apparel design, garment manufacturing technology, computer graphics and mathematics as a fundamental discipline [24].

Accordingly, this paper describes a part of the performed comprehensive study covering all stages of a complex computer preparation of patterns, anthropometric measurements carried out on a sample of male test subjects of a targeted age group using a 3D body scanner, computer-based $3 \mathrm{D}$ simulations of garment models prototypes, investigation of the impact of mechanical fabric properties [25], and simulation parameters for the model visualization. As the most complex part of the study a systematic series of activities aimed at linking the results of the individual segments and their implementation in the process of translating cutting patterns from a vector into a parametric format. In this sense, a systematic analysis and pattern preparation was made and mathematical expressions were defined whose use on the computer platform in the final stage allows the computer to adjust the pattern according to anthropometric characteristics of the individual body.

This part of the research represents a significant milestone in the research field of computer adaptations of patterns according to individual anthropometric characteristics, and by review of previously published scientific research papers in this area, no paper has been found that would be based on the presented concept.

\section{Methodology of the Garment Parametric Pattern De- velopment}

Transformations of patterns using the conventional 2D CAD system are performed by transformations of individual points of the pattern contours segments. In doing so, the implementation of transformations of one pattern does not depend on the other pattern, and thus in computer processing of patterns each pattern should be separately processed. The application of the innovative 2D/3D CAD system designed for $2 \mathrm{D} / 3 \mathrm{D}$ design and simulation of clothing allows you to perform the simultaneous modification of several patterns, which is most important for the 
adaptation of patterns, but the user needs to pre-determine the development steps which will allow the translation of $2 \mathrm{D}$ patterns from a vector into a parametric form [26].

\subsection{Construction, Modelling and Grading 2D Pattern of a men's Jacket}

By use of the Optitex 2D/3D CAD system for the garment construction preparation the development of the 2D pattern of a men's jacket model was performed whereby the patterns were computer graded for the selected sets of sizes. A constant value of ease for the pattern in the chest girth as the basic measurement for men's outerwear according to the size system used in the Republic of Croatia was defined. With regard to the deviation from normal proportions for the range of sizes from 54 to 60, which are primarily related to the measurement of waist circumference, a proportional increase in the coefficient of ease was made in this section. The verification of grading a men's jacket was made using the computer simulation of limited sizes on modified parametric models of the men's body Fig. 1, where all necessary parameters to perform simulations were previously determined Fig. 2 [17].
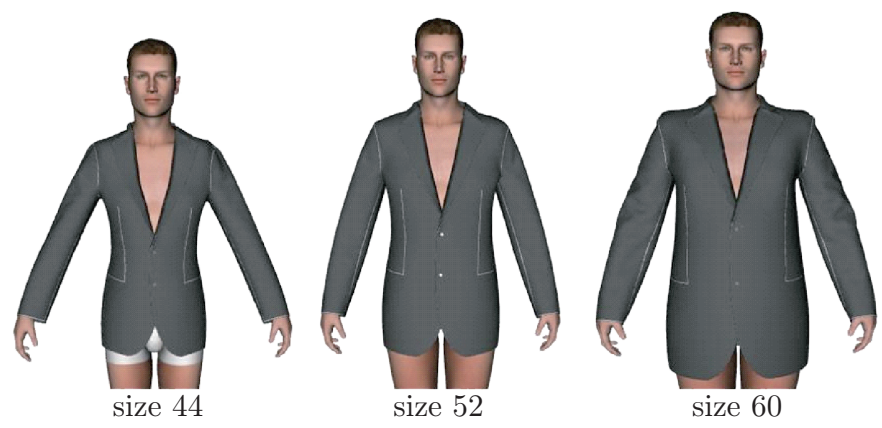

Fig. 1: Verification of grading limited sizes

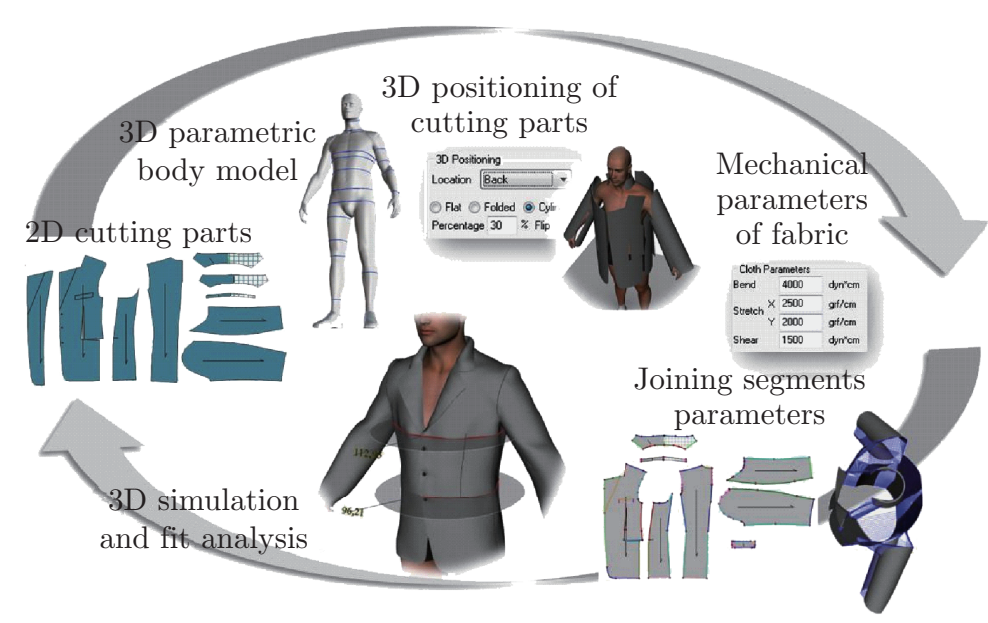

Fig. 2: Verification of garment pattern using 3D computer simulation 


\subsection{Determination of Development Steps for Translating Vector 2D Patterns into a Parametric form}

When defining segments of cutting patterns on which changes will be made, it is necessary to apply the knowledge of regularities and rules of conventional garment construction, anthropometric relationships of certain parts of the human body and deviations from normal body proportions. In this sense, the definition of segments of cutting patterns, on which changes will be made, depends on a garment model. Cutting patterns should be computer-adapted to the measurements of an individual, so it is necessary to make a complex analysis of a garment model pattern. Based on the principles of conventional garment construction and experience factors in the construction of custom-made clothing, it is necessary to predict segments on the cutting patterns that need to provide additional adjustments, depending on the body type where adjustments will be made. Accordingly, it is necessary to make the systematic development of a pattern in order to translate vector $2 \mathrm{D}$ cutting patterns into a parametric format suitable for the computer and automatic adjustment of measurements, while changes are defined for specifically selected segments and relationships of changes in corresponding segments and measurements on cutting patterns [23].

In this sense, it is initially necessary to determine the positions of fixed points, with which each cutting pattern will be fixed in the selected points. The positions of fixed points in the pattern determine the direction of segment movements during the adaptation of dimensions to the required body measurement. It is also necessary to define horizontal and vertical axes on all cutting patterns, where in the symmetrical patterns the vertical axis behaves as the axis of proportionality in relation to which modifications of enlarging or narrowing the cutting pattern for the coefficient of proportionality will be made. Horizontal axes are placed on the lines of the characteristic circumferences on the pattern, and if needed, at other horizontal lines, depending on the model pattern. The horizontal axes are linked to the points of the segments. They pass through and form the interdependence between connecting points and segments, where the connecting points are placed. After defining the vertical and horizontal axes it's necessary to define different types of measurements on flat and curve segments of cutting pattern. The vertical measurements connect segment points that are determined by the characteristic dimensions of the body height. The horizontal measurements are usually placed between the points connected with the horizontal axes, while the measurements on the curves are usually dependent on the previously placed vertical, horizontal and diagonal measurements. Each measurement should be given a name, and by defining the same name the measurements on the segments of the same or different cutting patterns and the dimensions of these segments are brought into interdependence, which allows simultaneous modification of all related segments of the pattern contour. Another way to introduce the relationship between the selected measurements on the cutting patterns can be achieved by establishing the ratio which allows to calculate the relationship between the measurements and to ensure the ratio proportionality of dimensions during adjustments. In this manner the cutting pattern is translated into parametric form, which can be tested by moving the interactive segment that is defined by a particular measurement. There is no unified rule to place the measurements on cutting patterns, as it is specific for each garment and is directly dependent on the rules of the conventional construction of each garment. Defining and linking the measurements depend on the anthropometric characteristics of the structure of certain body types which will be adjusted according to measurements, given the diversity of the dimensions of corresponding anthropometric measurements for different body figures.

As described above, the complete development of cutting patterns of men's jacket model was 
performed in the Modulate program, separately for three different men's body figure. In the next step it was necessary to determine mathematical expressions for specific body measurements on the basis of which recalculations of specific sets of predetermined measurements is aimed at achieving a specific value of the selected measurement. Axes and measurements are placed on the half of cutting patterns for which it was predefined that they are in a pair Fig. 4.

\subsection{Determination of Mathematical Expressions to Connect Measure- ments Sets on Cutting Patterns}

The most complex part of the research was finding mathematical expressions, which are necessary for calculating the dimensions of individual components of each cutting pattern. This is to ensure that in case of the modification of an individual cutting pattern all corresponding cutting patterns are changed to the same degree or for the specific coefficient of proportionality [23].

For the purpose of finding a mathematical expression, a number of measurements on cutting patterns were predefined. It is necessary to add together several values separately in order to define measurements of the pattern at the chest or waist line Fig. 3.

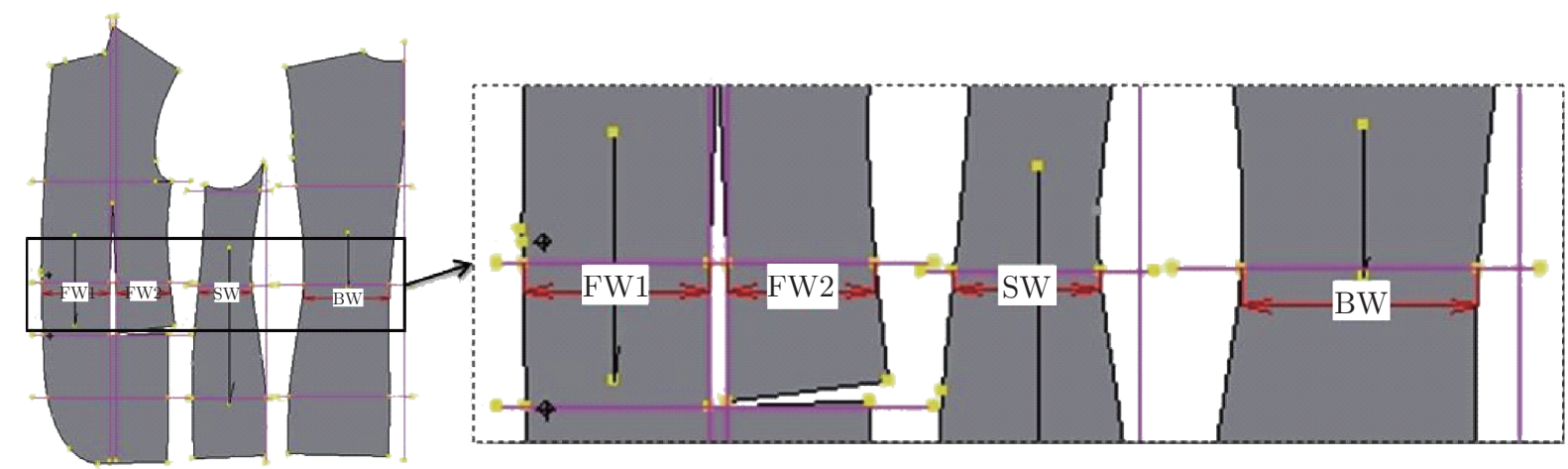

FW1 - front waist 1; FW2-front waist 2; SW- side waist; BW-back waist; WG-waist girth

Fig. 3: Set of measurements at the waist line

The obtained dimensions of characteristic range of the cutting pattern are to be reduced by the coefficient of ease of the pattern in order to ensure the proportionality of ease in case of a pattern adjustment according to the given body measurement. The values of all determined measurements at the waist line are listed in Table 1.

In addition, it is necessary to predict and determine the coefficients for the adjustment of individual measurements percentage shares in the total volume. This factor greatly depends on anthropometric characteristics of body types for which adjustments of cutting patterns will be made at a later stage due to the disproportionate increase in the characteristic circumferences of the human body for certain body types. In this sense, an analysis of cross-sections of typical circumferences of body models obtained with computer 3D scanning was performed and based on the performed analysis relationships of changes on individual segments depending on the characteristics of a body figure were defined Fig. 5.

Cross-section analysis of abdominal circumference showed disproportionate increase in the waist and abdominal area of full figure. By comparing cross-sections with inscribed ellipse and marking values of predefined measurements from cutting parts, calculation of percentage shares of 

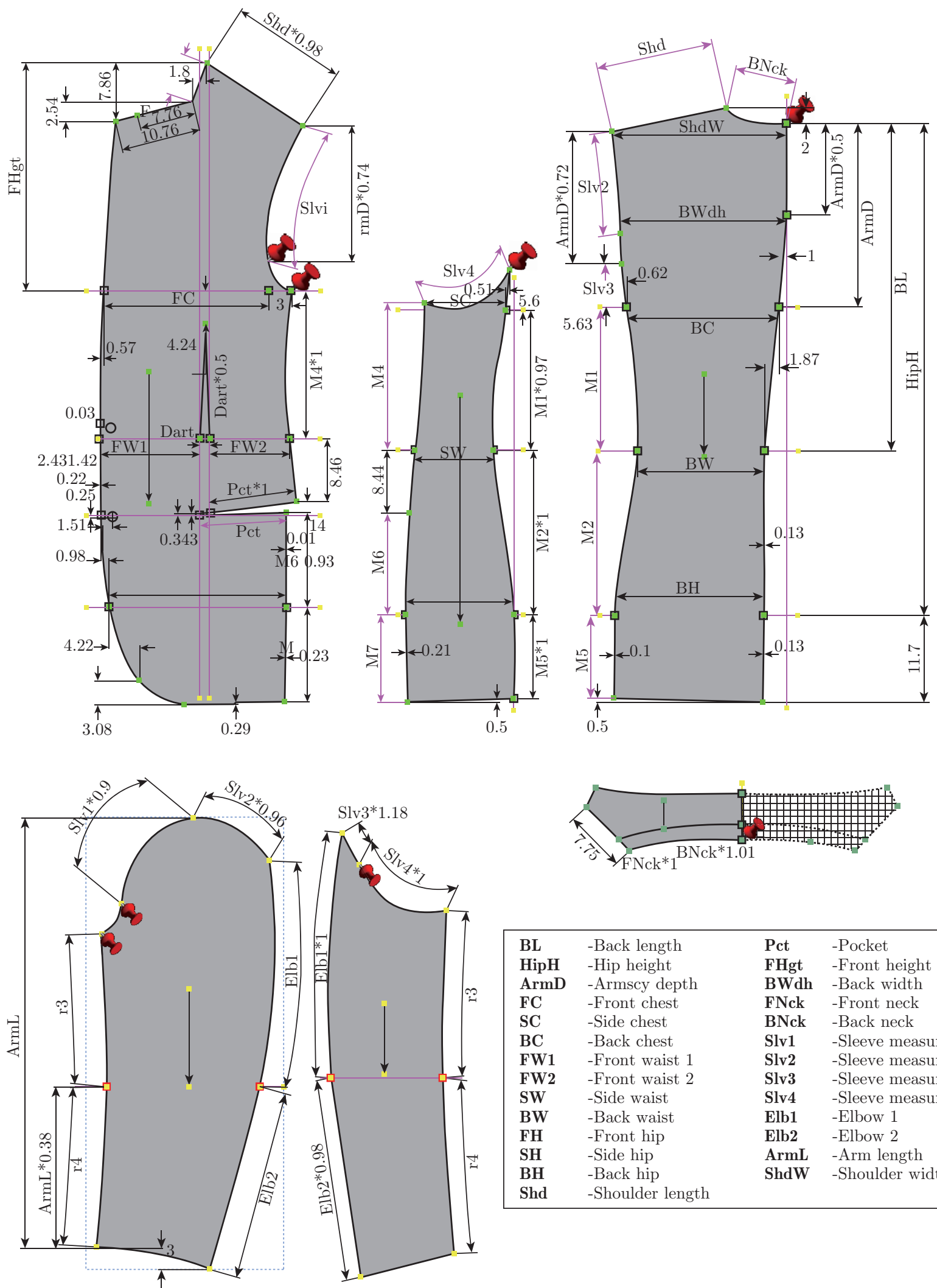

\begin{tabular}{|llll|}
\hline BL & -Back length & Pct & -Pocket \\
HipH & -Hip height & FHgt & -Front height \\
ArmD & -Armscy depth & BWdh & -Back width \\
FC & -Front chest & FNck & -Front neck \\
SC & -Side chest & BNck & -Back neck \\
BC & -Back chest & Slv1 & -Sleeve measure 1 \\
FW1 & -Front waist 1 & Slv2 & -Sleeve measure 2 \\
FW2 & -Front waist 2 & Slv3 & -Sleeve measure 3 \\
SW & -Side waist & Slv4 & -Sleeve measure 4 \\
BW & -Back waist & Elb1 & -Elbow 1 \\
FH & -Front hip & Elb2 & -Elbow 2 \\
SH & -Side hip & ArmL & -Arm length \\
BH & -Back hip & ShdW & -Shoulder width \\
Shd & -Shoulder length & & \\
\hline
\end{tabular}

Fig. 4: Cutting pattern of a men's jacket with systematically connected parameters (fixed points, axes and measurements) 
Table 1: Dimensions of cutting segments that define waist girth

\begin{tabular}{ccccccccccccc}
\hline Size & FW1 & $\%$ & FW2 & $\%$ & SW & $\%$ & BW & $\%$ & $\begin{array}{c}\text { WG } \\
\text { (garment) }\end{array}$ & $\begin{array}{c}\text { WG } \\
\text { (body) }\end{array}$ & $\begin{array}{c}\text { Ease } \\
\text { allow. }\end{array}$ & $\begin{array}{c}\text { Ease } \\
\text { allow. } \\
\text { coef. }\end{array}$ \\
\hline 44 & 10.1 & 11.48 & 9.4 & 10.68 & 9.0 & 10.23 & 15.5 & 17.61 & $\mathbf{8 8 . 0}$ & 78 & 10.0 & 0.886 \\
46 & 10.7 & 11.63 & 9.8 & 10.65 & 9.5 & 10.33 & 16.0 & 17.39 & $\mathbf{9 2 . 0}$ & 82 & 10.0 & 0.891 \\
48 & 11.3 & 11.77 & 10.2 & 10.63 & 10.0 & 10.42 & 16.5 & 17.19 & $\mathbf{9 6 . 0}$ & 86 & 10.0 & 0.896 \\
50 & 11.9 & 11.90 & 10.6 & 10.60 & 10.5 & 10.50 & 17.0 & 17.00 & $\mathbf{1 0 0 . 0}$ & 90 & 10.0 & 0.900 \\
52 & 12.4 & 11.92 & 11.1 & 10.67 & 11.0 & 10.58 & 17.5 & 16.83 & $\mathbf{1 0 4 . 0}$ & 94 & 10.0 & 0.904 \\
54 & 13.2 & 12.01 & 11.6 & 10.60 & 11.7 & 10.72 & 18.3 & 16.67 & $\mathbf{1 0 9 . 5}$ & 99 & 10.5 & 0.904 \\
56 & 13.9 & 12.09 & 12.1 & 10.52 & 12.5 & 10.86 & 19.0 & 16.52 & $\mathbf{1 1 5 . 0}$ & 104 & 11.0 & 0.904 \\
58 & 14.7 & 12.16 & 12.6 & 10.46 & 13.2 & 10.99 & 19.8 & 16.39 & $\mathbf{1 2 0 . 5}$ & 109 & 11.5 & 0.905 \\
60 & 15.4 & 12.22 & 13.1 & 10.40 & 14.0 & 11.11 & 20.5 & 16.27 & $\mathbf{1 2 6 . 0}$ & 114 & 12.0 & 0.905 \\
\hline
\end{tabular}

FW1 - front waist 1 FW2 - front waist 2 SW - side waist $\quad$ BW - back waist $\quad$ WG - waist girth
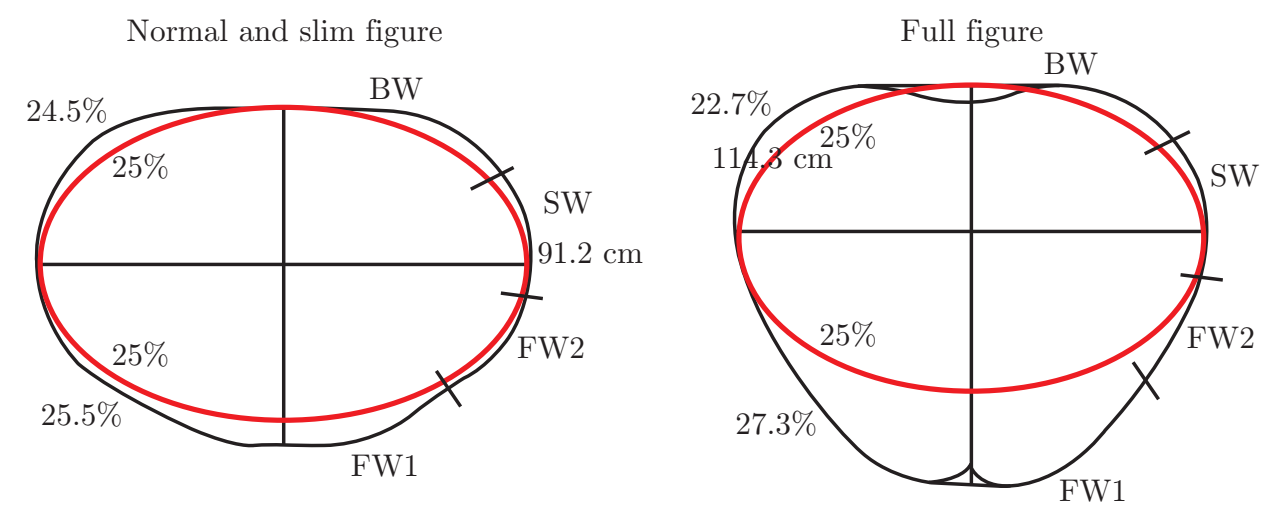

Fig. 5: Analysis of the abdominal circumference cross-section for normal, slim and full figure

correspondent segments shown that the side measures of the pattern can stay the same in its percentage and that on the account of back waist part, front waist should be increased.

When defining mathematical expressions for waist girth adjustment on slim figure, design visual body figure correction was made. Ease allowance of $10 \mathrm{~cm}$ on waist line, defined for normal and full figure, emphasize the waist with smaller amount of ease in relation to the chest where that amount is $12 \mathrm{~cm}$. Because of a big difference in body chest and waist girth on slim body figure, ease allowance on waist line was increased through adjustments of each separate measure percentage share by calculating coefficient of ease.

Taking into account all these influences and values of individual parameters, mathematical expressions for calculating values of individual measurements on cutting patterns were defined. Below are presented mathematical expressions and calculations for waist girth adjustment on slim and full figure.

$$
(\mathrm{FW} 1+\mathrm{FW} 2+\mathrm{SW}+\mathrm{BW}) \mathrm{x} 2=\mathrm{WG} 1=\text { garment waist girth }
$$

Slim figure: $\mathrm{WG} 1=(12 \% \mathrm{WG}+10,5 \% \mathrm{WG}+10,5 \% \mathrm{WG}+17 \% \mathrm{WG}) \mathrm{x} 2=(12+10,5+10$, 
$5+17) \times 2=100 \mathrm{~cm}$

Full figure: $\mathrm{WG} 1=(15 \% \mathrm{WG}+10,5 \% \mathrm{WG}+10,5 \% \mathrm{WG}+14 \% \mathrm{WG}) \times 2=(18,9+13,2+13$, $2+17,6) \times 2=126 \mathrm{~cm}$

$$
\mathrm{WG}=\mathrm{WG} 1 \mathrm{x} \text { ease allowance coefficient }=\text { body waist girth }
$$

Slim figure: $\mathrm{WG}=100 \times 0,904=90 \mathrm{~cm} \rightarrow$ ease allowance $=10 \mathrm{~cm}$

$\mathrm{WG}=100 \times 0,860=86 \mathrm{~cm} \rightarrow$ ease allowance $=14 \mathrm{~cm}$

Full figure: $\mathrm{WG}=126 \times 0,904=114 \mathrm{~cm}$

Since the system for pattern adjustments uses graded cutting patterns, adjustment according to the specified main body measurement is performed at the closest selected garment size. Defining the main body measurement depends on the desired type of adjustment, and the chest girth is defined for normal and slim figure, while the waist girth is defined for a fuller figure. For each garment size it is also necessary to define the range of selected measurement, according to which the nearest body size will be selected, depending on the last target dimension of measurement.

\subsection{Computer-based 3D Body Scanning and Linking the Obtained Data with the CAD System for Pattern Adjustments According to Individual Measurements and Garment Simulation}

A Vitus Smart laser 3D body scanner Human Solution and the accompanying program package ScanWorks V 2.7.2 was used for scanning, computer analysis and anthropometric measurements of a sample of 50 male test subjects with different anthropometric characteristics aged between 25 to 35 years. According to the defined measurements of parametric jacket patterns based on the results obtained by 3D scanning, a set of 10 body measurements was selected. For each test subject files of measurements in a format appropriate for entry of measurements data were generated. Automatic adjustment of the parametric pattern dimensions according to individual measurements of test subjects was performed by import of measurement file obtained by 3D body scanning. Parametric pattern of a men's jacket was adjusted in the selected measurements: chest girth, waist girth, hip girth, shoulder width, back width, armscye depth, back length, hip height, front height and arm length.

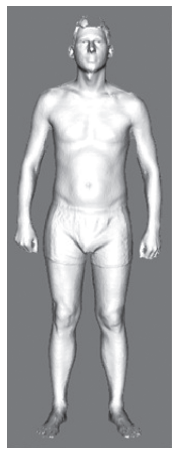

(a)

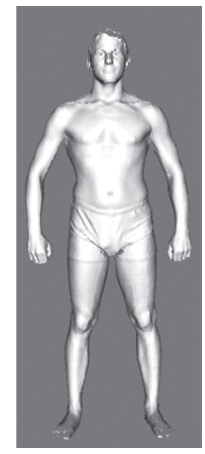

(b)

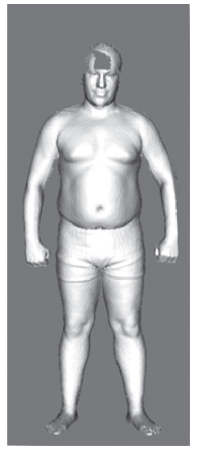

(c)

Fig. 6: Representation of the scanned 3D body models in the CAD system for garment simulation: a) tall stature and normal figure, b) normal stature and slim figure, c) short stature and full figure 
As a basis for the computer-based 3D simulation of parametric patterns adjusted to the measurements of test subjects the scanned computer 3D body models of test subjects were converted into a format suitable for the entry into the 3D CAD system for simulations as shown in Fig. 6. In case of such a direct entry of the body model obtained by 3D scanning as well as in case of the entry of a body model made by any commercial system for 3D modelling it is necessary to consider the compatibility of positioning the local coordinate system within the 3D objects and units of measurement used by a specific system.

\section{Results}

The verification of the determined expressions and testing the fit of the adjusted parametric cutting patterns was carried out with computer-based 3D simulations of adjusted patterns on computer-based models based on test subject bodies obtained through 3D scanning and a systematic analysis of the results in the sense of an objective assessment of the parametric garment model fit Fig. 7. For the purpose of fit assessment an analysis of pattern ease was performed by intersecting the visualized 3D body model and garment using transverse planes. The values of the body circumference and the model of the targeted cross-section were found, and the pattern ease was found from the difference of the values. Also, the control of the characteristic lengths of the model was performed by interactive measuring of the segment lengths of the 3D garment model. Furthermore, a computer-based analysis of fabric strain by zones of the garment model was performed for the purpose of the assessment of pattern fit and ease.

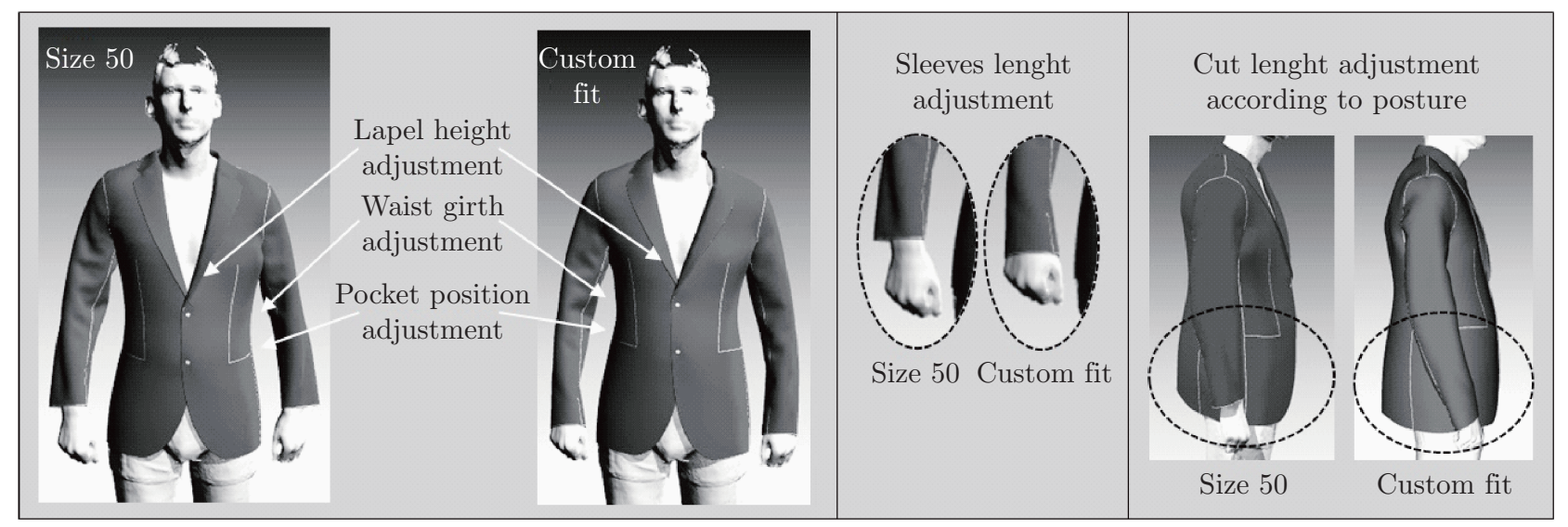

Fig. 7: Comparison of the pattern fit of a men's jacket adjusted according to the measurements of test subjects and the pattern of the corresponding garment size obtained by grading

\section{Conclusion}

Through linking 3D scanner determination of anthropometric body characteristics and the development of a computer-based parametric garment model of a men's jacket, a concept of pattern adjustments according to individual measurements for industrial manufacturing conditions was made. Considering the differences in garment models, there is no single rule that could be applied to each model, instead it is necessary to analyze each pattern separately. For this reason, the 
determination of measurements that will be changed in the pattern and finding mathematical expressions for calculating these changes is the hardest part of preparing patterns for subsequent adjustments. Fit assessment is also the verification of the success of the complete process, particularly the extensive preparation of cutting patterns in the Modulate program and applied mathematical expressions. The use of innovative technologies greatly assists in the implementation and achievement of quality results across all stages of development shown; however, it requires the application of new knowledge, based on the scientific research findings, continuous work and systematic combining knowledge from different fields.

The presented concept of the development of a parametric garment model can be regarded as one of the possible directions of development in the field of innovative computer technologies and 2D/3D CAD system in real industrial conditions. The results are important for clothing design and the product development cycle because the virtual prototype allowed us to visualize the wearing effects of the garments without actually producing sample garments. In according to this, the number of samples in the real product development cycle will be reduced. The next step in the investigation and further development of presented concept will be to focus on the development of parametric garment prototypes in accordance of the different body types. In this sense mathematical expressions defined for filler figure will be verified on a bigger sample of male test subjects. Testing sample will be increased in terms of age and number of participants as well as for athletic population considering their specific muscular body shape. Obtained data will be used for verification of present parametric garment model and for possible modifications of parametric pattern according to defined specific body characteristics of increased testing sample. Also, when defining mathematical expressions for different body types design aspect will be taken in consideration in terms of visual correction of body figure. This way pattern will not only ensure good dimensional fit but will also ensure appropriate design according to body type.

\section{References}

[1] Stylios GK, Wan TR, Powell NJ. Modelling the dynamic drape of garments on synthetic humans in a virtual fashion show. Int J Cloth Sci Tech 1996; 8: 95-112.

[2] Tae JK, Sung MK. Development of three-dimensional apparel CAD system: Part II: Prediction of garment drape shape. Int J Cloth Sci Tech 2000; 12: 39-49.

[3] Rödel, H, et al. Links between design, pattern development and fabric behaviours for clothes and technical textiles. Int J Cloth Sci Tech 2001; 13: 217-227.

[4] Cohen I, Li H. Inference of Human Postures by Classification of 3D Human Body Shape. AMFG '03 Proceedings of the IEEE International Workshop on Analysis and Modelling of Faces and Gestures, IEEE Computer Society Washington, DC, USA (C)2003. p. 74-83.

[5] Fan J, Yu W, Hunter L. Clothing appearance and fit: Science and Technology. Woodhead Publishing Limited and The Textile Institute, Cambridge England, 2004.

[6] Wang CCL. Parameterization and parametric design of mannequins. CAD 2005; 37: 83-98.

[7] Azouz ZB, Rioux M, Shu C, Lepage R. Characterizing human shape variation using 3D anthropometric data. Visual Comput 2006; 22: 302-314.

[8] Meng Y, Mok PY, Jin X. Interactive Virtual Try-on Clothing Design Systems. CAD 2010; 42: 310-321.

[9] Li J, Ye J, Wang Y, Bai L, Lu G. Fitting 3D garment models onto individual human models. Comput Graph 2010; 34: 742-755. 
[10] Liu YJ, Zhang DL, Yuen MMF. A survey on CAD methods in 3D garment design. Comput Ind 2010; 61: 576-593.

[11] Jiang J, Zhong Y, Wang S. 3D Mesh Deformation Technology for Generation of Virtual Garments. Journal of Fiber Bioengineering and Informatics 2011; 4: 129-136.

[12] Cui H, Wang R, Li Y. Parameterized Model for Virtual Human Deformation. Journal of Fiber Bioengineering and Informatics 2011; 4: 371-381.

[13] Petrak, S, Mahnic M, Rogale D, Ujevic D. Computer Design of Textile and Clothing Collection - Assumption of Contemporary Remote Business. Proceedings of $11^{\text {th }}$ World Textile Conference AUTEX 2011, Ecole Nationale Superieure d Ingenieurs Sud-Alace, Mulhouse France, 2011. p. 1162-1168.

[14] Simmons K, Istook CL, Devarajan P. Female figure identification technique (FFIT) for apparel Part II: Development of shape sorting software. JTATM 2004; 4: 1-15.

[15] Wang Y, Huang H. Three Dimensional Pattern Grading Based on Deformable Body Features and 3D Developable Surface. Journal of Fiber Bioengineering and Informatics 2011; 4: 115-128.

[16] Yang Y, Zou F, Ji X. A Case Study on Developing Virtual Dress Form Based on Body Shape Classification. Journal of Fiber Bioengineering and Informatics 2011; 4: 177-186.

[17] Ujević D, Petrak S, Hrastinski M, Mahnic M. Development of the garment size system and computer-based body models. Journal of Textiles and Engineer 2012; 19: 35-40.

[18] Petrak S, Mahnic M, Ujevic D. Study of the Computer-based Adjustment of a 3D Body Model Based on Anthropometric Data Obtained by 3D Laser Scanner. Proceedings of the 3rd International Conference on 3D Body Scanning Technologies, D'Apuzzo N. editor, Lugano, Switzerland, 2012. p. $115-126$.

[19] Lee SE, Chen JC. Mass-customization: Methodology for an Apparel Industry with a Future. Journal of Industrial Technology 1999; 16 information on http: //nait.org/jit/Articles/leee1222.pdf.

[20] Li J, Lu G. Customizing 3D garments based on volumetric deformation. Comput Ind 2011; 62: 693-707.

[21] Zülch G, Koruca HI, Börkircher M. Simulation-supported change process for product customization - A case study in a garment company. Comput Ind 2011; 62: 568-577.

[22] MacCarthy B, Brabazon PG, Bramham J. Fundamental modes of operation for mass customization. Int J Prod Econ 2003; 85: 289-304.

[23] Menga Y, Mok PY, Jin X. Computer aided clothing pattern design with 3D editing and pattern alteration. CAD 2012; 44: 721-734.

[24] Petrak S., Mahnić M. Implementation of modern computer systems in the process of industrial design and garment construction. Proceedings of $5^{\text {th }}$ Scientific-professional Symposium Textile Science and Economy, TZG, Faculty of Textile Technology, Zagreb Croatia, 2012. p. 25-36.

[25] Geršak J. Mehanske in fizikalne lasnosti tekstilnih materialov. Univerza v Mariboru, Fakulteta za strojništvo - založništvo, Maribor, 2006.

[26] Foley VD, Feiner H. Computer Graphics, Principles and Practice. Addison-Wesley, Workingham, 1990. 